Conclusions With the exception of one large network, RDS was not a particularly efficient way to screen for Ct/GC. Only one-fourth of those initially recruited by research staff in turn referred their social and sexual contacts. While social network testing has been adopted in the HIV testing realm, in the Ct/GC screening realm a focus on messages encouraging those who are tested to get their friends tested may have the greatest public health impact.

\section{S14.5 TREPONEMA PALLIDUM $\sigma 24$ REGULON AND ENVELOPE STRESS RESPONSE}

doi:10.1136/sextrans-2011-050102.60

L Giacani, 0 Denisenko, M Tompa, B Molini, A Centurion-Lara. University of Washington, Seattle, Washington, USA

Background During syphilis infection, the envelope of Treponema pallidum is constantly exposed to the host environment and, therefore, the most likely target of the host defences against the invading spirochaete. The mechanisms that maintain $T$ pallidum envelope integrity and functionality, particularly in response to host-induced stresses, are however poorly understood, and their elucidation would likely help identify important pathogenesis-associated molecules, perhaps related to T pallidum's ability to persist in the host despite a robust immune response. We hypothesised that in T pallidum, similarly to other Gram-negative pathogens, the transcription factor $\sigma 24$ ( $\sigma 24$, encoded by the rpoE gene, TP0092) might be a key element in maintaining $T$ pallidum envelope homeostasis. Putative $\sigma 24$ binding motifs can be identified in silico upstream of several $T$ pallidum genes that were experimentally shown to be involved in envelope stress response (ESR) in Escherichia coli. Furthermore, during early experimental syphilis $\sigma 24$ is highly transcribed compared to other $\sigma$ factors, and its expression increases even more as primary infection progresses. We therefore decided to investigate the possible role of $\sigma 24$ in T pallidum ESR by identifying the components of the T pallidum $\sigma 24$ regulon.

Methods $T$ pallidum cells grown in rabbits were fixed after harvest to crosslink DNA-binding proteins to their target sequences in the chromosome. DNA sequences recognised by $\sigma 24$ in vivo were isolated using chromatin immunoprecipitation in combination with high-throughput DNA sequencing (ChIP-seq) to identify bound DNA regions.

Results Thirty-nine DNA fragments targeted by $\sigma 24$ were identified in the $T$ pallidum chromosome. Seven of these target genes (lon-1, greA, ftsZ, prfB, htrA, and rpoE) were previously reported to be induced in response to envelope stress in $E$ coli, suggesting that the $T$ pallidum $\sigma 24$ regulon is likely to be similar to that of other bacteria. Other putative target genes encode transporters, cell division proteins and a subset of motility and chemotaxis proteins.

Conclusions In $T$ pallidum, $\sigma 24$ seems to control genes involved in a variety of cellular processes, including maintenance of envelope homeostasis and barrier function. Additional putative $\sigma 24$ dependent functions, apparently not directly involved in ESR, could as well be important in helping $T$ pallidum adapt to the host environment during the infection.

\section{S14.6 PREVALENCE AND PREDICTORS OF TRICHOMONAS INFECTION IN INCARCERATED WOMEN}

doi:10.1136/sextrans-2011-050102.61

${ }^{1} \mathrm{~A}$ Nijhawan, ${ }^{2} \mathrm{R}$ Salloway, ${ }^{3} \mathrm{~S}$ Andrea, ${ }^{4} \mathrm{~J}$ Champion, ${ }^{2} \mathrm{M}$ Seadale, ${ }^{2,5,6} \mathrm{~K}$ Chapin, 2,6J G Clarke. ${ }^{1}$ Harvard Medical School, Boston, Massachusetts, USA; ${ }^{2}$ Memorial Hospital of Rhode Island, Pawtucket, USA; ${ }^{3}$ Rhode Island Hospital, Providence, USA; ${ }^{4}$ Brown University, Providence, USA; ${ }^{5}$ Miriam Hospital, Providence, USA; ${ }^{6}$ Alpert School of Medicine at Brown University, Pawtucket, USA

Background Trichomonas vaginalis is the most prevalent curable sexually transmitted infection in the United States and may lead to preterm delivery, infertility and increased HIV transmission. Illicit drug use, HIV infection and Black race have been associated with high rates of infection. Incarcerated women may be at especially high risk for infection, though few studies have examined routine screening for trichomonas in this population.

Methods Women $>18$-years-old entering the Rhode Island Department of Corrections between September 2009 and December 2010 were recruited to participate. All women submitted a self-collected vaginal swab for trichomonas culture and Transcription Mediated Amplification testing. Each participant completed a survey addressing demographics, symptoms, sexual behaviour, and substance use by audio computer-assisted self-interview. Data analysis was completed using multivariate logistic regression in STATA.

Results 288 women enrolled in the study, mean age was 28 years. $59 \%$ of participants were White, $17 \%$ Hispanic, $12 \%$ Black and $12 \%$ other races. Forty-three per cent reported vaginal symptoms and $54 \%$ reported illicit drug use in the 30 days prior to incarceration. Among all participants, the prevalence of trichomonas was $8.7 \%$ by culture and $12.5 \%$ by NAAT. The strongest predictors of infection included Black race (OR 4.1, 95\% CI 1.4 to 12.0), cocaine use in the 30 days prior to incarceration (OR 2.5, 95\% CI 1.1 to 5.7 ), and $>3$ year since last pap smear (OR 5.2, 95\% CI 1.5 to 17.8). Vaginal symptoms and age were not significantly associated with trichomonas detection.

Conclusions Trichomonas infection is common in incarcerated women, especially among Blacks, recent cocaine users and those not receiving routine gynaecologic care. Infection was not predicted by symptoms or by age. Routine screening for trichomonas infection in high-risk populations may lead to increased detection and treatment.

\section{S15 STI epidemiology in Europe: challenges for prevention and control \\ S15.1 SEXUALLY TRANSMITTED INFECTIONS IN EUROPE: COORDINATING THE EUROPEAN STI NETWORK}

doi:10.1136/sextrans-2011-050102.62

M van de Laar. European Centre for Disease Prevention and Control, Stockholm, Sweden

Background Since 2008, the European Centre for Disease Prevention and Control is coordinating the enhanced STI surveillance in $30 \mathrm{EU} /$ EEA countries. Each country was requested to nominate experts for collaboration and data submission to the European Surveillance System. Five STI are under surveillance, syphilis, congenital syphilis, gonorrhoea, chlamydia and LGV, as per Decision 2119/98/EC of the European Commission.

Methods Surveillance objectives and the set of variables for enhanced STI surveillance were agreed upon in the annual network meeting and training session to use the European Surveillance System for data submission. Data were collected for the period 1990-2009; two network meetings were organised for all $30 \mathrm{EU} /$ EEA countries to discuss the preliminary results.

Results Chlamydia is the most frequently reported STI in Europe, accounting for the majority of all STI reports with 343958 cases in 2009 (185 per 100000 population). Chlamydia was reported more in women than in men and $75 \%$ were reported in young people (15 and 24 years). Chlamydia is increasing continuously over time. In 2009, 29202 gonorrhoea cases have been reported (9.7/100 000) and nearly a quarter of all gonorrhoea cases were reported in MSM. For syphilis, 18317 cases have been reported (4.5 per 100000$)$ and half of syphilis cases were reported in MSM. The overall trend in gonorrhoea and syphilis across the EU/EEA showed a notable decreasing trend in 
countries which previously had reported very high rates. These declines are probably due to changes in healthcare systems, diagnostic capacity and reporting rather than true changes in the incidence. However, dramatic increases were noted in other countries and-based on the information from the male-to-female ratio-this is most likely due to recent increases of syphilis among MSM. The overall trend in chlamydia showed a continuously increasing trend, reflecting an increase in testing and screening practices across countries. These trends must be interpreted with caution due to the heterogeneity in reporting and healthcare systems. A further limitation to the interpretation is that many diagnoses are either not made or under-reported. Diagnoses from certain countries cannot be included in trend analyses as they do not have comprehensive surveillance for STI.

Conclusion Enhanced surveillance of STI in Europe is essential to provide the information that is necessary to monitor the distribution of disease and to evaluate the public health response to control the transmission of infections. Collaborating within the European STI expert networks provides the platform for sharing best practices and expert knowledge across Europe.

\section{S15.2 EUROPEAN GONOCOCCAL ANTIMICROBIAL SURVEILLANCE PROGRAMME (EURO-GASP): TOWARDS TIMELIER MONITORING}

doi:10.1136/sextrans-2011-050102.63

${ }^{1} \mathrm{M} J$ Cole, ${ }^{1} \mathrm{~S}$ A Chisholm, ${ }^{2} \mathrm{M}$ Unemo, ${ }^{3} \mathrm{~S}$ Hoffmann, ${ }^{4} \mathrm{M} \mathrm{J}$ W van de Laar, ${ }^{1} \mathrm{C}$ A Ison. ${ }^{1}$ Health Protection Agency, London, UK; ${ }^{2}$ Örebro University Hospital, Örebro, Sweden; ${ }^{3}$ Statens Serum Institut, Copenhagen, Denmark; ${ }^{4}$ European Centre for Disease Prevention and Control, Stockholm, Sweden

Background It is imperative that antimicrobial resistance (AMR) surveillance systems work in a timely manner and outcomes are translated into treatment guidelines without delay. For the first time in 2010, the European gonococcal antimicrobial surveillance programme (Euro-GASP) introduced biannual testing to allow more frequent reporting of trends and developments in Neisseria gonorrhoeae AMR across Europe.

Methods In the first half of 2010, 18 out of the 30 EU/EEA Member States contributed up to 55 consecutive gonococcal isolates to EuroGASP. Susceptibility testing was performed by Etest or agar dilution for therapeutically relevant antimicrobials: azithromycin, cefixime, ceftriaxone, ciprofloxacin, gentamicin and spectinomycin. Biannual testing continued into the second half of 2010. Additionally 10 laboratories performed decentralised testing with the submission of AMR data into Euro-GASP instead of gonococcal isolates.

Results In the first round of biannual testing $900 \mathrm{~N}$ gonorrhoeae isolates were tested. Eight per cent of the isolates displayed decreased susceptibility $(>0.125 \mathrm{mg} / \mathrm{l})$ to cefixime, which was an increase from $5 \%$ in 2009 . No decreased susceptibility to ceftriaxone was detected. For ciprofloxacin, the overall incidence of resistant isolates decreased from $63 \%$ in 2009 to $52 \%$ in 2010 and azithromycin resistance decreased from $13 \%$ in 2009 to $5 \%$ in 2010 . No resistance to spectinomycin was demonstrated. The MIC50 and MIC90 of gentamicin in the isolates remained at $8 \mathrm{mg} / \mathrm{l}$ in 2010 .

Conclusions The results from the first round of biannual testing have shown that isolates displaying decreased susceptibility to cefixime are rapidly increasing. This is a major concern as cefixime is a widely used, recommended therapy for gonorrhoea across Europe. Healthcare professions should be aware of cefixime treatment failures, particularly in light of the recent identification of treatment failures in Norway and the UK. The MICs of ceftriaxone need to be monitored continually to preserve this precious treatment option. Euro-GASP is currently being adjusted to allow for timely reporting and decentralised testing including representativeness and links to epidemiological data. All of which contribute to monitoring the ever changing gonococcus in timelier and more efficient ways, with the ultimate aim of treating infected individuals successfully.

\section{S15.3 EUROPEAN MSM INTERNET SURVEY (EMIS): DIFFERENCES IN SEXUALLY TRANSMISSIBLE INFECTION TESTING IN EUROPEAN COUNTRIES}

doi:10.1136/sextrans-2011-050102.64

${ }^{1} \mathrm{U}$ Marcus, ${ }^{1} \mathrm{~A} J$ Schmidt, ${ }^{2} \mathrm{M}$ Breveglieri, ${ }^{3} \mathrm{P}$ F Davila, ${ }^{3} \mathrm{~L}$ Ferrer, ${ }^{3} \mathrm{C}$ Folch, ${ }^{4} \mathrm{~F}$ Hickson, ${ }^{5} \mathrm{H} J$ Hospers, ${ }^{2} \mathrm{M}$ Mirandola, ${ }^{4} \mathrm{D}$ Reid, ${ }^{4} \mathrm{P}$ Weatherburn, for the EMIS network. ${ }^{1}$ Department for Infectious Disease Epidemiology, Robert Koch Institute, Berlin, Germany; ${ }^{2}$ Regional Centre for Health Promotion, ULSS 20-Veneto Region, Verona, Italy; ${ }^{3}$ Centre for Epidemiological Studies on HIVIAIDS in Catalonia (CEEISCAT), Barcelona, Spain; ${ }^{4}$ Sigma Research, London School of Hygiene and Tropical Medicine, UK; ${ }^{5}$ Maastricht University, University College Maastricht, The Netherlands

Background Comparing rates of sexually transmissible infections (STIs) among men who have sex with men (MSM) in different European countries is challenging due to national differences in reporting systems, healthcare systems, infectious disease surveillance methods, quality of data, and/or levels of social acceptance of homosexual behaviours and openness about homosexuality.

Methods From June through August 2010, the European MSM Internet Survey (EMIS) mobilised more than 180000 respondents from 38 European countries to complete an online questionnaire in one of 25 languages. The questionnaire covered sexual happiness, HIV and STI-testing and diagnoses, unmet prevention needs, intervention performance, HIV-related stigma and gay-related discrimination. Recruitment was organised predominantly online, through gay social media, and links and banners on more than 100 websites for MSM all over Europe.

Results Perceptions on access to free/affordable STI-testing differed across Europe (median: 80\%; range: $40-95 \%$ ); and was substantially correlated with reported recent STI-testing $\left(R^{2}=0.27\right)$. Quality of STI-testing was highly diverse: While blood-testing was common in all participating countries, only Ireland, Malta, and the UK seem to offer penile or particularly anal examinations as standard of care. In all participating countries HIV-positive respondents reported higher rates of both STI-testing and diagnosis. Self-reported STI-screening among men without HIV diagnosis ranged from 10\% (Turkey) to $37 \%$ (Netherlands). Substantial correlations between rates of testing procedures appropriate for MSM (such as anal or genital swabs) and diagnosed gonorrhoea $\left(R^{2} H I V-p o s=0.24\right)$ or Chlamydia infections were observed $\left(R^{2} H I V\right.$-pos $=0.50 ; R^{2}$ others $\left.=0.29\right)$.

Conclusion Self-reported testing and diagnosis rates for bacterial STIs suggest high levels of under-diagnosis and unmet sexual healthcare needs in most European countries. In Europe, there is an urgent need to implement or improve sexual healthcare tailored to MSM-specific needs.

\section{S15.4 RE-EMERGENCE OF LYMPHOGRANULOMA VENEREUM IN EUROPE AND THE PUBLIC HEALTH RESPONSE}

doi:10.1136/sextrans-2011-050102.65

${ }^{1} \mathrm{H}$ Ward, ${ }^{2} \mathrm{H} \mathrm{J} \mathrm{C}$ de Vries, ${ }^{3} \mathrm{M}$ van de Laar. ${ }^{1}$ Imperial College, London, UK; ${ }^{2} \mathrm{GGD}$ Amsterdam, The Netherlands; ${ }^{3}$ European Centre for Disease Prevention and Control, Stockholm, Sweden

Background In January 2004, public health officials in the Netherlands noted an outbreak of cases of lymphogranuloma venereum (LGV) among men who have sex with men (MSM). Since then a number of outbreaks and cases have been reported from European countries, North America and Australia. The re-emergence of LGV in 